\title{
Expectativas de las mujeres en el periodo posnatal
}

\author{
Women's expectations in the postnatal period
}

\section{Comentado de:}

Finlayson K, et al. PLOS One 2020, 22;15:e 0231415, PMID: 32320424. doi: 10.1371/journal.pone.0231415 ${ }^{1}$

\section{Objetivo}

Identificar las perspectivas, expectativas y percepciones de las mujeres en el periodo posparto a fin de mejorar la atención brindada por los servicios posnatales y satisfacer las necesidades de las mujeres, sus familias y sus recién nacidos.

\section{Diseño}

Revisión sistemática y síntesis temática de estudios cualitativos.

\section{Población}

Fueron elegibles para esta revisión los estudios cualitativos que incluyesen relatos en primera persona de mujeres y neonatos sanos, independientemente de la paridad, el tipo de parto y el entorno. Fueron excluidos aquellos estudios que únicamente investigaron las experiencias en relación a la provisión de cuidado posnatal, temáticas específicas como la lactancia, la alimentación infantil, los cuidados neonatales críticos, ciertas condiciones patológicas, y los estudios de casos, las tesis o los resúmenes de conferencias.

\section{Estrategia de búsqueda y selección de estudios}

Los autores realizaron la búsqueda en base al formato PEO (población, exposición y desenlace o outcome) para identificar estudios cualitativos en diversas bases de datos como CINAHL, MEDLINE, PsycINFO, EMBASE, Global Index Medicus, POPLINE, Revistas Africanas en Línea y LILACS, publicados desde 2003 hasta mayo de 2019. Dos autores analizaron de manera independiente la elegibilidad de los resúmenes.

\section{Extracción y análisis de los datos}

La extracción de datos se realizó en base al principio de síntesis temática. Los artículos fueron analizados mediante codificación y mapeo, elaborando un mapa conceptual de los resultados de la revisión, llegando luego a un acuerdo de todos los autores sobre el mapa conceptual final. El nivel de confianza de los hallazgos de la revisión fue evaluado mediante la herramienta de Grade CERqual.

\section{Resultados}

Se incluyeron 36 estudios de moderada y alta calidad, realizados en 15 países con más de 800 mujeres.

La mayoría de los datos se habían recopilado mediante entrevistas individuales y/o grupos focales o de discusión.

El análisis de los estudios condujo a la conformación de cinco temas analíticos, que se presentan en la Tabla 1.

\section{Conclusiones}

La evidencia hallada sostiene que el rol de la familia y de la comunidad informada, educada y activa es fundamental para que las mujeres reciban información realista y apropiada, ya que la misma facilita la experiencia posparto positiva.

Fuente de financiamiento/Conflicto de interés de los autores: El estudio fue encargado a la Universidad de Central Lancashire por un programa de investigación patrocinado por distintas entidades (como UNICEF y el Banco Mundial) y ejecutado por la Organización Mundial de la Salud. Los financiadores no tuvieron ningún rol en el diseño del estudio, la recopilación y análisis de datos, la decisión de publicar, o la escritura del manuscrito.

Los autores no informaron conflictos de interés.

Tabla 1. Hallazgos principales en los temas identificados como de interés para las mujeres en el periodo posparto

\begin{tabular}{|c|l|}
\hline Tema analítico & \multicolumn{1}{c|}{ Resumen de resultados } \\
\hline $\begin{array}{c}\text { Cabalgando los } \\
\text { "rápidos" emocionales }\end{array}$ & $\begin{array}{l}\text { Las mujeres presentan emociones conflictivas y contradictorias en el periodo posparto, desde momentos de dicha y feli- } \\
\text { cidad intensas, hasta sensación aguda de soledad, tristeza y depresión. Estas últimas emociones pueden generar senti- } \\
\text { miento de culpabilidad y falta de confianza en su capacidad como madres, que pueden agravarse por la fatiga. }\end{array}$ \\
\hline $\begin{array}{c}\text { Bailando alrededor del } \\
\text { bebé - adaptación } \\
\text { social y relacional }\end{array}$ & $\begin{array}{l}\text { Los ajustes emocionales y psicológicos se deben, al menos en parte, a cambios abruptos de las viejas rutinas por otras } \\
\text { nuevas. Las mujeres priorizan el cuidado del bebé, y se les dificulta la realización de otras actividades. El énfasis en el } \\
\text { bebé puede ejercer presión sobre las relaciones de pareja; cambios en la libido pueden generar ansiedad adicional. }\end{array}$ \\
\hline $\begin{array}{c}\text { Se requiere de una } \\
\text { comunidad para criar } \\
\text { a una madre }\end{array}$ & $\begin{array}{l}\text { Las mujeres valoran el apoyo práctico y emocional que reciben de sus parejas, familiares y, en algunos contextos, ancianas } \\
\text { de la comunidad; así como el entendimiento y consejos brindados por otras madres. Los profesionales de la salud también } \\
\text { pueden desempenar un papel a la hora de ofrecer tranquilidad, validación y orientación. }\end{array}$ \\
\hline $\begin{array}{c}\text { Rearmando el cuerpo } \\
\text { del parto }\end{array}$ & $\begin{array}{l}\text { Las mujeres no se sienten preparadas para los efectos físicos y psicológicos del parto (p. ej. posibilidad de complicacio- } \\
\text { nes luego del parto o la cesárea); y algunas quisieran tener mayor diálogo e información de los profesionales de salud. } \\
\text { Presentan dificultad en adaptarse al nuevo cuerpo posparto, y a inseguridades generadas por la expectativa social sobre } \\
\text { cambios en el peso, lo cual también puede afectar su deseo sexual. }\end{array}$ \\
\hline $\begin{array}{c}\text { Colocando a la madre } \\
\text { en atención posnatal }\end{array}$ & $\begin{array}{l}\text { Las mujeres quieren ser vistas como individuos y sentir que sus necesidades son reconocidas y satisfechas al igual que } \\
\text { las de sus bebés. Sin embargo, pueden encontrarse con rutinas hospitalarias no adaptadas a estas necesidades y sentir } \\
\text { que el soporte que se les brinda es inadecuado. }\end{array}$ \\
\hline
\end{tabular}




\section{Comentario}

La revisión resumida sintetiza la información de estudios cualitativos de distintos continentes y de más de 800 mujeres evaluando perspectivas, expectativas y percepciones en torno a qué cosas le importan a las mujeres en el posparto, con el fin de conocer las necesidades y las dificultades que ellas atraviesan en este periodo para incorporar su perspectiva en el desarrollo de las guías de cuidado. Los autores explican que el documento logra retratar la complejidad de esta temática, dado que la mayoría de los estudios incluidos tenían un nivel de confianza alto y moderado de acuerdo a la herramienta CERqual. Una de las limitaciones de la evidencia aportada por esta revisión sistemática es la falta de estudios realizados en poblaciones de países de bajos recursos.

La reformulación de la pregunta PICO (población, intervención, control y desenlace o outcome) en los estudios de metasíntesis cualitativa es útil para poder conjugar los distintos modelos pragmáticos y trans-teóricos de los estudios cualitativos, logrando así la esquematización y la posibilidad de mayor inclusión de artículos para su análisis posterior. Sin embargo, existen líneas críticas a este tipo de estudio que argumentan que este enfoque pierde la individualidad y la singularidad de la mirada cualitativista, ya que se realiza un recorte de información que no logra transmitir la profundidad de la temática. No obstante, las revisiones sistemáticas de estudios cualitativos son pilares de evidencia en los cuales se pueden basar los entes reguladores y los organismos internacionales para construir guías de cuidado y desarrollar políticas públicas.

La mayor importancia del artículo resumido radica en que intenta poner en agenda internacional la temática del puerperio con una visión ampliada y realista, que considera las valoraciones de las mujeres que transitan este periodo. Asimismo, aporta información relevante para la práctica diaria, brindando un abordaje diferente al clásico médico-biológico.

\section{Conclusiones de la comentadora}

La evidencia hallada sostiene que la provisión de información adecuada y el acompañamiento activo del sistema de salud, la familia y la comunidad es fundamental para mejorar la experiencia posparto de las mujeres. Asimismo, se necesitan más estudios de países de bajos y medianos ingresos para ampliar esta mirada.

Maria Belen Doval [ Servicio de Medicina Familiar y Comunitaria, Hospital Italiano de Buenos Aires. maria.doval@hospitalitaliano.org.ar ]

Doval MB. Expectativas de las mujeres en el periodo posnatal. Evid Actual Pract Ambul. 2021;24(3):e002142. Available from: https://dx.doi.org/10.51987/ EVIDENCIA.V24I4.6968. Comentado de: Finlayson K, et al. PLOS One 2020, 22;15:e 0231415, PMID: 32320424, doi: 10.1371/journal.pone.0231415

\section{Referencias}

1. Finlayson K, Crossland N, Bonet M, et al. What matters to women in the postnatal period: A meta-synthesis of qualitative studies. PLOS One. 2020;15(4):e0231415. Available from: doi:10.1371/journal.pone.0231415. 